\title{
Rating Scales for Assessing Infection Responses of Barley Infected with Cochliobolus sativus
}

\author{
Thomas G. Fetch, Jr., and Brian J. Steffenson, Department of Plant Pathology, North Dakota State University, \\ Fargo 58105
}

\begin{abstract}
Fetch, T. G., Jr., and Steffenson, B. J. 1999. Rating scales for assessing infection responses of barley infected with Cochliobolus sativus. Plant Dis. 83:213-217.

Spot blotch, caused by Cochliobolus sativus, is a common foliar disease of barley that is controlled primarily through the deployment of resistant cultivars. Resistance is often assessed at the seedling and adult plant stages, but currently no comprehensive visual scale exists that describes the full spectrum of infection responses (IRs) occurring on barley. From the evaluation of a diverse collection of barley germ plasm and C. sativus isolates, a 1 to 9 IR scale was developed based on the type (presence of necrosis and chlorosis) and relative size of spot blotch lesions observed on the second leaves of barley seedlings. The nine IRs were classified into three general categories of low (IRs 1 to 3), intermediate (IRs 4 and 5), and high (IRs 6 to 9) hostparasite compatibility. Low IRs consisted of minute to small necrotic lesions with no or very slight diffuse marginal chlorosis. Intermediate IRs consisted of medium-sized necrotic lesions with a distinct but restricted chlorotic margin, while high IRs consisted of large necrotic lesions with distinct chlorotic margins and varying degrees of expanding diffuse chlorosis. In addition to the seedling IR scale, a four-class adult plant IR scale $(\mathrm{R}=$ resistant, $\mathrm{MR}=$ moderately resistant, $\mathrm{MS}=$ moderately susceptible, and $\mathrm{S}=$ susceptible) was developed based again on the type and relative size of lesions present on the leaves. These rating scales should be useful for many types of studies on spot blotch of barley.
\end{abstract}

Additional keywords: disease assessment, Hordeum vulgare, host-parasite genetics, resistance

Cochliobolus sativus (Ito \& Kuribayashi) Drechs. ex Dastur (anamorph: Bipolaris sorokiniana (Sacc.) Shoem.), the causal agent of spot blotch, is a common foliar pathogen of barley (Hordeum vulgare L.) in the Upper Midwest region of the United States and the central Canadian provinces (14). Yield losses ranging from 16 to $33 \%$ have been reported for susceptible barley cultivars (2). Although fungicides can be effective in reducing spot blotch severity (7), the most effective and environmentally sound means of control is through the use of resistant cultivars. Resistance in barley to $C$. sativus is often evaluated at the seedling stage in the greenhouse and at the adult plant stage in the field $(4,5,11,15)$. In classifying the disease reactions of barley lines for breeding purposes or for studies in host-parasite genetics, it is important to have a rating scale that describes fully the range of possible infection responses (IRs) encoun-

\section{Corresponding author: T. G. Fetch, Jr. \\ E-mail address: fetchjr@prairie.nodak.edu}

Accepted for publication 4 December 1998.

Publication no. D-1999-0122-01S

(C) 1999 The American Phytopathological Society tered. For spot blotch of barley, Cook (3) devised a scale with five classes to distinguish qualitative and quantitative differences of lesions on seedlings. Over a 9year period (1990 to 1998), we characterized the lesion type polymorphisms of several thousand barley lines to a diverse collection of $C$. sativus isolates and discovered distinct IRs that were not described in the scale developed by Cook (3). Thus, the objective of this research was to develop a comprehensive IR rating scale based on the type (presence of necrosis and chlorosis) and relative size of lesions observed on barley seedlings infected with $C$. sativus. An IR rating scale also was developed for adult plants infected with $C$. sativus in the field. For each rating scale, color photographs were made of the different lesion types to aid in the classification of IRs by other researchers.

\section{MATERIALS AND METHODS}

Host genotypes and planting protocols. Several thousand barley lines from the North Dakota barley improvement programs, National Small Grains Collection (USDA-ARS Small Grains and Potato Germplasm Research Facility, Aberdeen, ID), and Steptoe/Morex doubled haploid population (17) were tested for their reaction to spot blotch in greenhouse and field trials from 1990 to 1998 at Fargo, North Dakota. In the greenhouse trials, two replicates (4 to 5 seeds per replicate) of each barley line were planted in plastic cones (20.7 $\mathrm{cm}$ deep and $3.8 \mathrm{~cm}$ diameter) or pots $(12.5 \mathrm{~cm}$ deep and $10 \mathrm{~cm}$ square) containing a $75 \%$ peat moss: $25 \%$ perlite potting mix. A controlled-release fertilizer (14-14-14, N-P-K) was added at a rate of $1.0 \mathrm{~g}$ per cone or $4.0 \mathrm{~g}$ per pot. The seeded potting mix was then watered with a watersoluble fertilizer (15-0-15, N-P-K, 536 ppm $\mathrm{N}$ rate). Plants were grown in a greenhouse at $23 \pm 2{ }^{\circ} \mathrm{C}$ with supplemental lighting provided by $1,000 \mathrm{~W}$ metal halide lights $\left(530\right.$ to $\left.710 \mu \mathrm{E} \cdot \mathrm{m}^{-2} \cdot \mathrm{s}^{-1}\right)$ for $14 \mathrm{~h} /$ day. The same barley lines also were planted in field nurseries to assess the IR at the adult plant stage. Lines were sown in hill plots (spaced $0.3 \mathrm{~m}$ apart, within and between rows) with a four-row cone planter. The hill plots were planted with the interior two cones of the planter, while the outside two cones were set to plant a continuous row of ND 5883, a two-rowed barley line that is highly susceptible to spot blotch. The rows of ND 5883 were planted to facilitate the spread of inoculum in the disease nursery.

Inoculum production and inoculation. Over the past 9 years at North Dakota State University (NDSU), we have investigated the lesion types exhibited by barley to a diverse collection of $C$. sativus isolates (56 total isolates). This $C$. sativus collection includes isolates from Brazil, China, Japan, New Zealand, Poland, Uruguay, and the United States. Of the 33 isolates from the United States, three (isolates ND85F [ATCC accession 201651], ND90Pr [ATCC accession 201652], and ND93-1 [ATCC accession 201653]) have been studied extensively. Isolate ND85F (pathotype 1 [23]) has been utilized in disease resistance screening tests of barley germ plasm at NDSU since 1986, and its virulence pattern is well-established. Isolates ND90Pr (pathotype 2 [23]) and ND93-1 (pathotype 0 [23]) also were used routinely in resistance screening tests because they exhibit virulence patterns that are different from each other and from isolate ND85F on the differential set of barley lines $(5,23)$. Isolates were maintained as conidia stored on silica gel crystals at $4^{\circ} \mathrm{C}(20)$. Inoculum was produced by aseptically transferring a few silica gel 
crystals with adsorbed conidia onto 9-cm petri plates containing yeast extract corn starch agar (YECSA) $\left(1.0 \mathrm{~g}\right.$ of $\mathrm{KH}_{2} \mathrm{HPO}_{4}$, $0.5 \mathrm{~g}$ of $\mathrm{MgSO}_{4} \cdot \mathrm{H} 2 \mathrm{O}, 4.0 \mathrm{~g}$ of Difco yeast extract, $15.0 \mathrm{~g}$ of corn starch, and $20 \mathrm{~g}$ of agar per liter of distilled water). The cultures were incubated at $21^{\circ} \mathrm{C}$ with a 12 -h photoperiod (20W fluorescent bulbs emitting 260 to $280 \mu \mathrm{E} \cdot \mathrm{m}^{-2} \cdot \mathrm{s}^{-1}$ ) for 10 days.

Inoculum was prepared, quantified, and applied to plants following the general protocols of Mumford (10) and Tuite (20). Conidia were harvested by adding distilled water to the plate and scraping the agar surface with a rubber spatula. This conidial suspension was filtered through two layers of cheesecloth to remove mycelial fragments (10). Hemacytometer counts of conidia provided estimates of the inoculum concentration (20). Additional volumes of distilled water were added to adjust the inoculum concentration to either 5,000 (greenhouse inoculation) or 8,000 (field inoculation) conidia per $\mathrm{ml}$. A surfactant (polyoxyethylene-20-sorbitan monolaurate) was added ( $100 \mu \mathrm{l}$ per liter) to the conidial suspension to facilitate dispersion of the inoculum over the leaf surfaces. Fourteenday-old seedlings (second leaf fully expanded) were inoculated with the conidial suspension at a rate of approximately 0.2 $\mathrm{ml}$ per plant. Our previous studies had shown this inoculum concentration and rate to be optimal for establishing discreet lesions on the leaves. Inoculum was applied using an atomizer (Model 15, DeVilbiss Co., Somerset, PA) pressurized by air from a pump at $60 \mathrm{kPa}(10)$. After inoculation, plants were placed in the dark in closed chambers $\left(20^{\circ} \mathrm{C}\right)$ maintained near saturation by periodic misting from ultrasonic humidifiers for $18 \mathrm{~h}$. The chamber doors were then opened to allow the plant surfaces to slowly dry. Plants were returned to the greenhouse under the same conditions described previously.

In the field, spot blotch epidemics were initiated by two methods. The first method involved placing barley straw infected with isolate ND85F at the base of the susceptible "spreader" row plants of line ND5883 (about $40 \mathrm{~g}$ of straw per linear $\mathrm{m}$ of row). The infected straw was from the previous year's experiment (e.g., straw from 1990 was used for the 1991 field experiment) and was placed in the nursery when most plants were at the mid-tillering stage of development (Zadoks growth stage [GS] 24) (24). The second method was by foliar inoculation of the conidial suspension (8,000 conidia per $\mathrm{ml}$ of isolate ND85F or ND90Pr) on barley test entries when the awns of most plants were emerging (Zadoks GS 49). This inoculum was applied at a rate of $48 \mathrm{ml}$ per $\mathrm{m}^{2}$ using a 1.2m-long $\mathrm{CO}_{2}$ pressurized aluminum spray boom $(5,12)$. Plants were inoculated during the evening when the environmental conditions were predicted to be favorable for dew formation.
Disease assessment. Seedling IRs were assessed on the second leaves of plants 10 to 12 days after inoculation, depending on the temperature during incubation. Adult plant IRs were assessed on the upper three leaves of plants at the early to mid-dough stage of development (Zadoks GS 83-85). The timing for IR assessment was made when the susceptible controls (ND 5883 and Bowman) were expressing fully susceptible lesions, but before complete tissue collapse occurred. The general size ranges of the necrotic and chlorotic (if present) zones of the lesions were measured with a ruler. Leaves exhibiting uniform lesion types were photographed and made into color plates to aid in the classification of IRs by other researchers.

\section{RESULTS}

Seedling infection response scale. A nine-class IR rating scale was developed based on the type (presence of necrosis and chlorosis) and relative size of lesions observed on barley lines infected with isolates of $C$. sativus (Fig. 1). Most of the described IRs were based on infection by isolate ND85F, but almost all of the other isolates tested (49 of 55) gave similar lesion types. The following seedling IR rating scale includes a general description (i.e., presence of necrosis or chlorosis, shape, and relative size) of the lesion types, a general range of lesion dimensions (including necrosis and chlorosis) for the IRs, and a color plate of each lesion type. IRs 1 and 2 consisted of minute round and small oblong necrotic lesions without chlorosis, respectively (Fig. 1-1 and -2). Lesions of these two IRs ranged from about 0.3 to $0.7 \mathrm{~mm}$ in length and 0.3 to 0.5 in width. IR 3 consisted of larger round to oblong necrotic lesions approximately 0.8 to $1.3 \mathrm{~mm}$ long and 0.5 to $0.7 \mathrm{~mm}$ wide with very slight $(<0.5 \mathrm{~mm}$ wide) diffuse marginal chlorosis (Fig. 1-3).

IRs 4 and 5 consisted of medium-sized elliptical to somewhat ovate necrotic lesions with distinct but restricted chlorotic margins (Fig. 1-4 and -5). These lesion types ranged from about 1.4 to $3.9 \mathrm{~mm}$ in length and 0.7 to $1.3 \mathrm{~mm}$ in width with 0.3 to $0.7 \mathrm{~mm}$ wide chlorotic margins. Generally, these lesions are two to three times longer and wider than those of IR 3.

IRs $6,7,8$, and 9 were similar in that all consisted of elliptical to elongated elliptical necrotic lesions with distinct chlorotic margins and expanding diffuse chlorosis (Fig. 1-6 to -9). The expanding diffuse chlorosis can be observed as the lighter colored tissue occurring between lesions on infected leaves (e.g., the left two-thirds versus the right one-third of the leaf in Fig. 1-6). Zones of the diffuse chlorosis usually extended longitudinally along the leaf, often joining adjacent lesions. IRs 6 to 9 were differentiated on the basis of relative lesion size. Individual lesions of these IRs ranged from about 4.0 to $8.0 \mathrm{~mm}$ in length and 1.4 to $3.2 \mathrm{~mm}$ in width with chlorotic margins ranging from 0.5 to $1.0 \mathrm{~mm}$ wide.

The nine IRs were classified into three general categories of low, intermediate, and high host-parasite compatibility. Minute to small necrotic lesions (with no or very slight diffuse marginal chlorosis) characteristic of IRs 1, 2, and 3 were considered indicative of low compatibility. Medium-sized necrotic lesions with a distinct but restricted chlorotic margin characteristic of IRs 4 and 5 were considered indicative of intermediate compatibility. Finally, large necrotic lesions with distinct chlorotic margins and varying degrees of expanding diffuse chlorosis characteristic of IRs $6,7,8$, and 9 were considered indicative of high compatibility.

In addition to the nine IRs described above, several other lesion types also were occasionally observed. One was a diffuse "blotchy" necrotic lesion. This diffuse necrotic reaction was characterized by irregular light to dark brown necrotic lesions ranging in size from about 1.0 to 7.0 $\mathrm{mm}$ in length and 1.0 to $2.0 \mathrm{~mm}$ in width (Fig. 2A). Another was a chlorotic halo reaction where small to medium-sized elliptical (about 0.5 to $3.0 \mathrm{~mm}$ in length and 0.5 to $1.0 \mathrm{~mm}$ in width) necrotic lesions were surrounded by large chlorotic halos (about 0.5 to $2.5 \mathrm{~mm}$ wide) (Fig. 2B). A third type consisted of lesions with distinct tan necrosis (Fig. 2C). These lesions were somewhat ovate, ranging from about 2.0 to $5.0 \mathrm{~mm}$ in length and 1.0 to $2.0 \mathrm{~mm}$ in width with restricted marginal chlorosis. Seedlings infected with isolate ND90Pr typically exhibited light brown necrotic lesions with whitish gray centers and marginal to expanding chlorosis in compatible reactions (Fig. 3A). These are distinctly different from the more common solid dark brown necrotic lesions induced by isolate ND85F (Fig. 3B) and most of the other $C$. sativus isolates evaluated. The lesions depicted in Figures $2 \mathrm{~B}$ and $\mathrm{C}$ and $3 \mathrm{~A}$ may be indicative of intermediate to high host-parasite compatibility, whereas those depicted in Figure 2A may be indicative of low host-parasite compatibility.

Adult infection response scale. Based on the lesion types observed on several thousand barley accessions in the field, an adult plant IR scale was developed (Fig. 4). Although the size of the lesions on leaves varied almost continuously, four general classes (resistant, moderately resistant, moderately susceptible, and susceptible) were distinguished based on the type (presence of necrosis and chlorosis) and relative size of lesions observed. Resistant (R) IRs were characterized by small ( $<2 \mathrm{~mm}$ long) round to oblong dark brown necrotic lesions, while moderately resistant (MR) IRs were characterized by mediumsized (2 to $4 \mathrm{~mm}$ long) elliptical dark brown necrotic lesions with no to slight diffuse marginal chlorosis. Moderately susceptible (MS) IRs consisted of medium 
to large (4 to $6 \mathrm{~mm}$ long) elliptical light brown necrotic lesions with marginal chlorosis, while susceptible (S) IRs consisted of large (>6 mm long) elongated elliptical light brown necrotic lesions with expanding areas of chlorosis. The same general IR classes were observed in response to both isolates $\mathrm{ND} 85 \mathrm{~F}$ and ND90Pr in the field. IRs of R and MR are considered indicative of low host-parasite compatibility, whereas IRs of MS and S are considered indicative of high hostparasite compatibility.

\section{DISCUSSION}

Rating scales for assessing the IRs of seedling and adult barley plants infected with $C$. sativus were developed based on the evaluation of a large and diverse set of host accessions and pathogen isolates. These IRs were classified on the basis of lesion type (presence of necrosis and chlorosis) and relative lesion size and are illustrated in color plates to aid in their classification by other researchers. The utility and value of IR rating scales can be realized as they are applied in various types of studies. In this regard, the seedling IR scale has proven valuable for classifying different lesion types in studies on the genetics of host resistance in barley to C. sativus $(17,21,22)$, genetics of virulence in $C$. sativus on barley (22), virulence di-

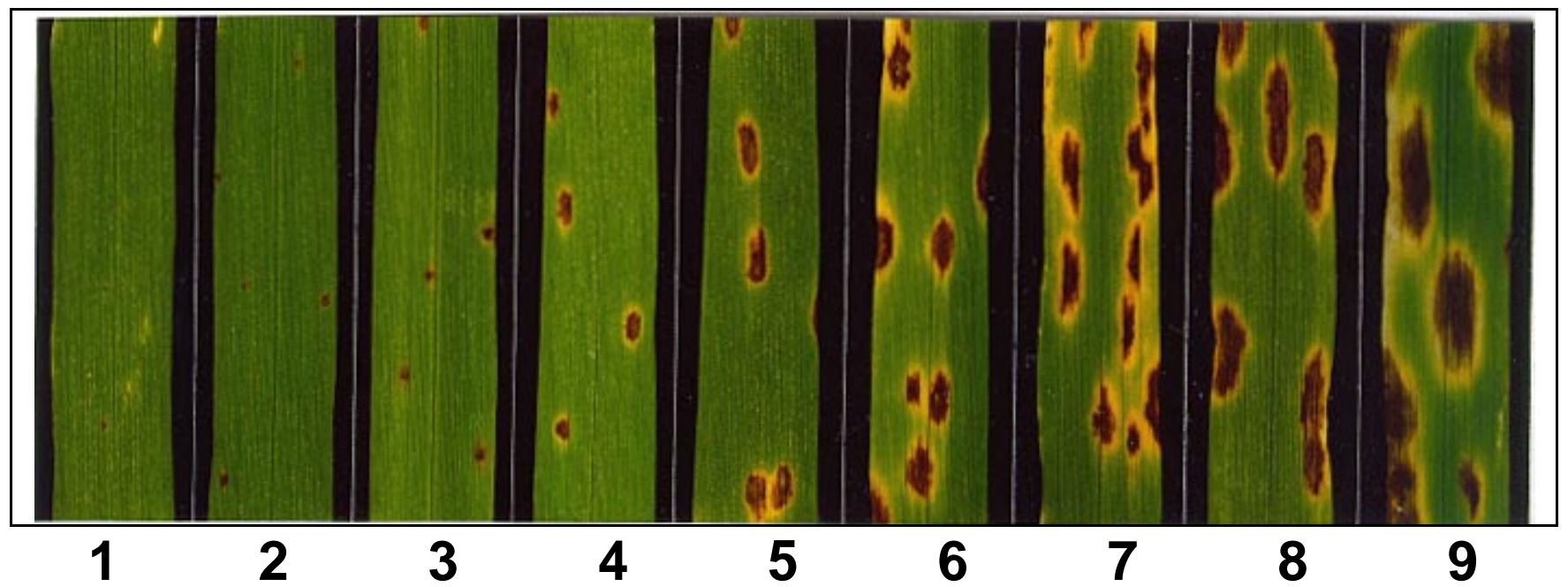

Fig. 1. An infection response (IR) rating scale for barley seedlings infected with Cochliobolus sativus. IRs were based on the type (presence of necrosis and chlorosis) and relative size of lesions observed on the second leaves of barley seedlings. The nine IRs were classified into three general categories of low, intermediate, and high host-parasite compatibility. Minute to small necrotic lesions (with no or very slight diffuse marginal chlorosis) characteristic of IRs 1, 2, and 3 were considered indicative of low compatibility. Medium-sized necrotic lesions with a distinct but restricted chlorotic margin characteristic of IRs 4 and 5 were considered indicative of intermediate compatibility. Large necrotic lesions with distinct chlorotic margins and varying degrees of expanding diffuse chlorosis characteristic of IRs $6,7,8$, and 9 were considered indicative of high compatibility. The leaves depicted in the color plates are $1.5 \times$ their original size.

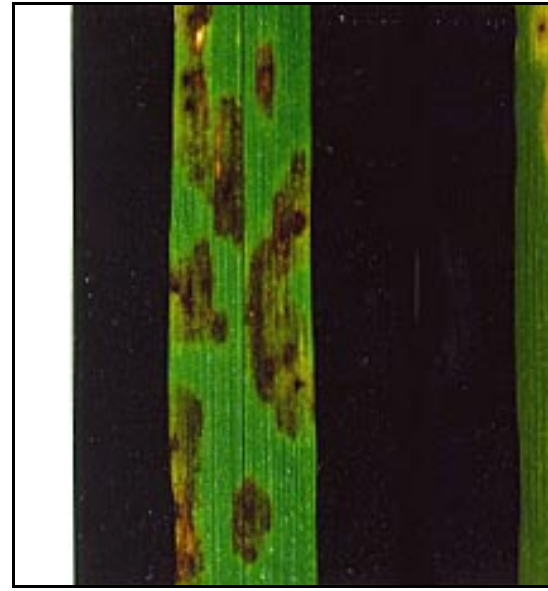

A

Fig. 2. Additional infection responses (IRs) observed on barley seedling leaves infected with Cochliobolus sativus: (A) diffuse necrotic reaction, which is characterized by irregular diffuse light to dark brown necrotic lesions of variable size; (B) chlorotic halo reaction, which is characterized by small to medium-sized elliptical necrotic lesions surrounded by large chlorotic halos; and (C) tan necrotic reaction, which is characterized by somewhat ovate lesions of variable size with distinct tan necrosis and restricted marginal chlorosis. The lesions depicted $\mathrm{B}$ and $\mathrm{C}$ may be indicative of intermediate to high host-parasite compatibility, whereas those depicted in A may be indicative of low host-parasite compatibility. The leaves depicted in the color plates are $1.5 \times$ their original size.

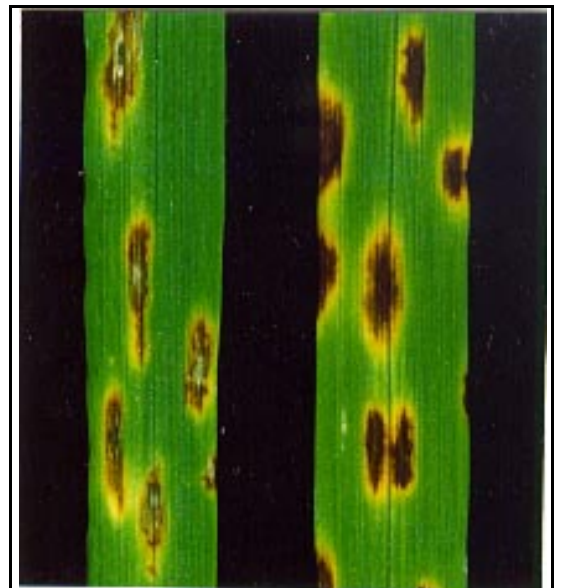

A

B

Fig. 3. Compatible infection responses (IRs) observed on barley seedlings infected with Cochliobolus sativus isolates $\mathrm{ND90Pr}(\mathbf{A})$ and $\mathrm{ND} 85 \mathrm{~F}$ (B). Isolate ND90Pr typically induces light brown necrotic lesions with whitish gray centers and marginal to expanding chlorosis on susceptible barley lines. These lesions are distinctly different from the more common solid dark brown necrotic lesions induced by isolate ND85F and most other $C$. sativus isolates on susceptible barley lines. Leaves in color plates are $1.5 \times$ their original size. 


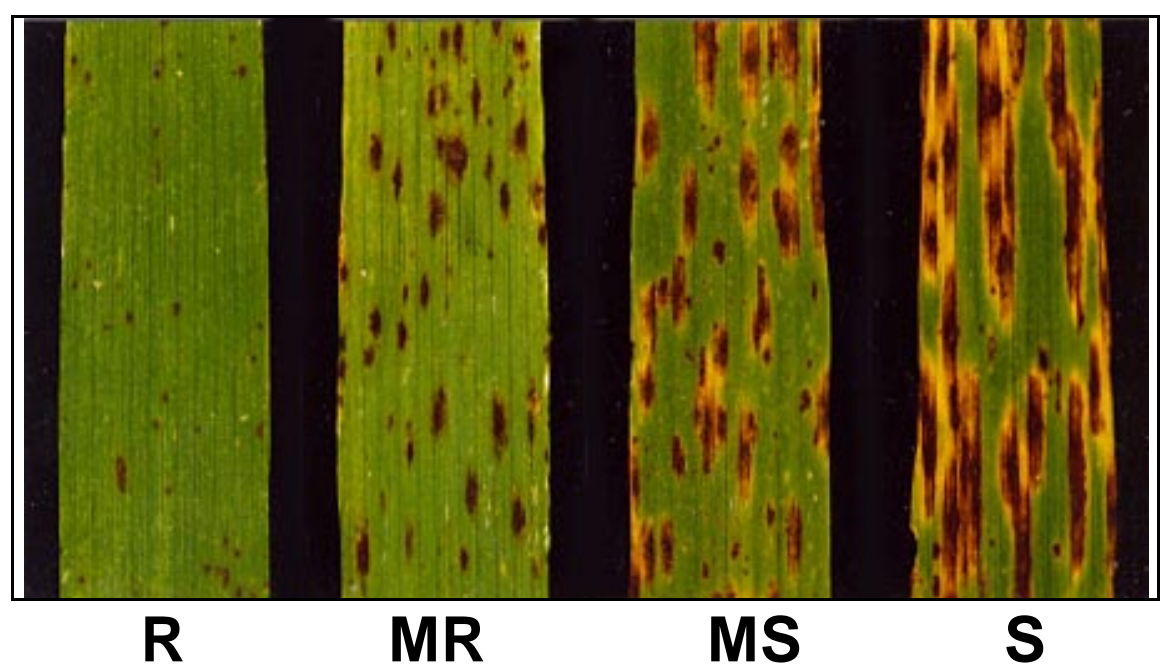

Fig. 4. An infection response (IR) rating scale for adult barley plants infected with Cochliobolus sativus. IRs were based on the type (presence of necrosis and chlorosis) and relative size of lesions observed on the upper three leaves of plants at the early to mid-dough stage of development. Resistant (R) IRs were characterized by small round to oblong dark brown necrotic lesions, while moderately resistant (MR) IRs were characterized by medium-sized elliptical dark brown necrotic lesions with no to very slight diffuse marginal chlorosis. Moderately susceptible (MS) IRs consisted of medium to large elliptical light brown necrotic lesions with marginal chlorosis, while susceptible (S) IRs consisted of large elongated elliptical light brown necrotic lesions with expanding areas of chlorosis. IRs of R and MR were considered indicative of low host-parasite compatibility, whereas IRs of MS and $\mathrm{S}$ were considered indicative of high host-parasite compatibility. The leaves depicted in the color plates are actual size.

versity in C. sativus (23), and selection of barley lines resistant to $C$. sativus (16). The adult plant IR rating scale also has proven valuable for assessing various lesion types on barley infected with $C$. sativus. The classification of these different lesion types using the IR scale facilitated the molecular mapping of a spot blotch resistance locus (B. Steffenson, unpublished) and the selection of spot blotch resistant lines in the field $(15,16)$.

In a previous study, Cook (3) developed a five-class rating scale diagram for assessing the IRs of barley seedlings to $C$. sativus. This scale was based on both qualitative and quantitative attributes of the lesions. While this scale was used successfully for detecting resistance in a limited number of breeding lines at NDSU (4), it did not include the full spectrum of lesion types that were observed in these evaluations. After the work of Cook (3), V. D. Pederson and his associates at NDSU developed 0 to 9 scales for assessing the spot blotch resistance of barley seedlings in the greenhouse and adult plants in the field (V. D. Pederson, personal communication). However, these scales were based on disease severity and did not discriminate among the different lesion types occurring on barley lines infected with various isolates of $C$. sativus. The IR scale developed in this study is primarily qualitative and based on lesion type. Quantitative aspects of lesion size are considered in this IR scale, but only in a relative or comparative sense (e.g., lesions of IR 8 are comparatively larger than lesions of IR 7). The lesions exhibited by barley to isolates of $C$. sativus probably vary almost continuously, especially for size. The IR scales developed in this study were designed to capture a large portion of this lesion variation in nine discreet IR classes for seedlings and four discreet IR classes for adult plants. The number of IR classes included in the scales is arbitrary; nevertheless, the described IR classes do encompass the full range of lesion type variation that we observed in our studies on a diverse collection of host accessions and pathogen isolates.

C. sativus is comprised of several distinct pathotypes that differ in their virulence on barley $(5,23)$. Most of the IRs described in this study were based on inoculations with isolate ND85F (pathotype 1 ), which has been used for a number of years to screen breeding lines for resistance to spot blotch at NDSU (15). Fortynine isolates (including those representing pathotypes 0 and 1 ) from different regions of the United States and the world (Brazil, China, Japan, New Zealand, Poland, and Uruguay) induced lesion types on barley seedlings that were similar to those induced by isolate ND85F (M. ValjavecGratian, T. Fetch, Jr., and B. Steffenson, unpublished). A notable exception was isolate ND90Pr (pathotype 2), which induced a distinctly different lesion type than isolate ND85F on barley seedlings. Isolate ND90Pr, plus five other pathotype 2 isolates, induced sunken whitish gray lesions during the early stages of infection (about $48 \mathrm{~h}$ postinoculation). Mature lesions (about 14 days postinoculation) typically were light brown with whitish gray centers and chlorotic margins (Fig. 3A). Thus, in addition to possessing a different virulence pattern (5), pathotype 2 isolates also induce a distinctly different lesion type on barley. Isolate ND85F usually induces a solid dark brown necrotic lesion with expanding chlorosis (the "classic" spot blotch lesion) in highly compatible interactions (Fig. 3B), but it occasionally induces a diffuse necrosis (Fig. 2A), chlorotic halo (Fig. 2B), or tan necrosis (Fig. 2C) reaction on some barley lines. The tan spot disease of wheat, caused by Pyrenophora tritici-repentis, has symptoms similar to those of spot blotch on barley (8). Isolates of this pathogen are known to produce necrosis and chlorosis toxins in wheat $(1,19)$. Additionally, wheat lines that differ in their reaction to one or both of these toxins have been reported (9). C. sativus also produces toxins $(6,13)$. The basis for the different lesion types observed in this study is not known, but may be related to the production of different pathogen toxins interacting with several resistance genes in the host. The role of $C$. sativus toxins in symptom expression on barley should be investigated further.

The expression of lesion types on barley seedlings infected with $C$. sativus can be influenced by a number of factors. The choice of leaf for inoculation is one important consideration for assessing spot blotch IRs. We found that infected primary leaves often exhibited larger and more variable lesion types than those on the second and third leaves. Additionally, infected primary leaves senesced more rapidly than infected second or third leaves. In our screening evaluations of barley germ plasm, we found that the second leaf was best for separating known resistant and susceptible lines. Thus, we recommend that IR ratings be based on lesions occurring on the second or (if necessary) third leaves. To properly assess the IRs of barley to $C$. sativus, it is critical that the lesions be well spaced and moderate in number on the leaves. High inoculum densities often produce lesions that coalesce, making it difficult to reliably assess the type and size of individual lesions. In contrast, low inoculum densities can result in insufficient numbers of lesions for assessing the IRs of barley lines. The inoculation protocols used in this study consistently produced sufficient numbers of well-separated lesions for assessing IRs on barley seedlings. Finally, the size of lesions can vary across a leaf depending on the position of the infection site. Lesions are often larger on the leaf tips and margins, and at the point where the second leaf bends downward (B. Steffenson and T. Fetch, unpublished). The reason for this is unknown. We recommend that the seedling IR be based on a sample of lesions from the central portion of the leaf blade, excluding the point at which the leaf bends downward.

In this study, approximate size range measurements were provided for the gen- 
eral lesion types. The given lesion dimensions were not intended to be a rigid or fixed criterion upon which the IRs are classified. Rather, they were given as supplemental information for describing and separating IR classes and for general comparison purposes by other researchers. We never intended, nor do we ever expect, any user of this scale to actually measure lesions for assessment of spot blotch IRs. It simply would not be practical to measure individual lesions for the routine evaluation of barley accessions for resistance to spot blotch. Instead, one can use the color plates as a guide to quickly and easily estimate the relative size of lesions present on barley lines as needed for the classification of the spot blotch IRs (especially IRs 6 to 9).

In the course of our research in the $H$. vulgare-C. sativus pathosystem, we found that many homozygous barley lines often display two (or rarely three) different but sequential IRs. For our disease evaluation notes, we list the most common IR first followed by the second most common type, with the third type (if present) listed in parenthesis, e.g., 3,2(4). Generally, the most common IR comprises over $75 \%$ of all IRs observed on an individual barley line. For the final presentation of data, we give the one (IR mode) or two most common IRs observed and the IR range (the lowest and highest IRs observed) $(5,23)$. These data provide inferences as to the central tendency and variability of the IRs observed.

The classification of plants into resistant and susceptible categories is a primary consideration in the selection of breeding lines with resistance and in studies on hostparasite genetics. For breeding purposes, we generally advance lines exhibiting IRs of 1 to 5 at the seedling stage and R to MR at the adult plant stage. Lines with these IRs will likely sustain less damage to the photosynthetic system and consequently have less yield loss (3). However, the final selection criterion will depend on the level of resistance desired in a barley cultivar. In several studies on the genetics of resistance in barley (17, B. Steffenson, unpublished), we used the same criterion (IRs 1 to $5=$ resistant and 6 to $9=$ susceptible) as for the screening of breeding lines. This criterion can change depending on a number of factors, including the level of resistance in the parental lines and the virulence of the pathogen isolate. For example, ValjavecGratian (21) classified plants exhibiting IRs 1 to 3 as resistant and those exhibiting IRs 4 to 9 as susceptible in an inheritance study of resistance in barley to $C$. sativus. Using this criterion, resistance (i.e., low compatibility) was found to be conferred by two genes in two different barley populations.

The correlation between the disease reaction at the seedling stage in the greenhouse and the adult plant stage in the field is an important consideration in programs breeding for disease resistance. With spot blotch of barley, some investigators have found a good correlation between the seedling and adult plant reaction $(4,10)$, whereas others have not (11). The recent results by Steffenson et al. (17) documenting the presence of loci that confer resistance at the seedling or adult plant stage indicate that researchers should not rely solely on the seedling reaction for assessing potential adult plant resistance. In the Upper Midwest, the adult plant reaction is of primary importance because spot blotch rarely reaches severe levels prior to the heading stage of barley. For these resistance evaluations, we assess both the IR and the disease severity (percent [0 to 100] leaf area affected by disease) of lines in the field (15).

Similar IR scales have been developed for the barley net blotch (18) and wheat tan spot diseases (8). These rating scales have been very useful in many types of studies in the respective pathosystems and have been widely used. The IR scales we developed have been valuable in many types of studies on spot blotch at NDSU and will likely be useful for other researchers investigating the $H$. vulgare-C. sativus pathosystem.

\section{ACKNOWLEDGMENTS}

We thank Vernyl D. Pederson, professor emeritus, for his valuable assistance in the photography of disease specimens.

\section{LITERATURE CITED}

1. Brown, D. A., and Hunger, R. M. 1993. Production of a chlorosis-inducing, host-specific, low-molecular weight toxin by isolates of $P y$ renophora tritici-repentis, cause of tan spot of wheat. J. Phytopathol. 137:221-232.

2. Clark, R. V. 1979. Yield losses in barley cultivars caused by spot blotch. Can. J. Plant Pathol. 1:113-117.

3. Cook, R. J. 1960. Studies on the reactions of resistant and susceptible barley leaves to Helminthosporium sorokinianum. M.S. thesis. North Dakota State University, Fargo.

4. Cook, R. J., and Timian, R. G. 1962. Lesion type as a means of detecting resistance in barley leaves to the spot blotch Helminthosporium. Phytopathology 52:1086-1089.

5. Fetch, T. G., Jr., and Steffenson, B. J. 1994. Identification of Cochliobolus sativus isolates expressing differential virulence on two-row barley genotypes from North Dakota. Can. J.
Plant Pathol. 16:202-206.

6. Gayed, S. K. 1962. The pathogenicity of six strains of Helminthosporium sativum to three cereals with special reference to barley. Mycopathologia 18:271-279.

7. Kiesling, R. L. 1985. The diseases of barley. Pages 269-308 in: Barley Agron. Monogr. 16. D. C. Rasmusson, ed. ASA and SSSA, Madison, WI.

8. Lamari, L., and Bernier, C. C. 1989. Evaluation of wheat lines and cultivars to tan spot (Pyrenophora tritici-repentis) based on lesion type. Can. J. Plant Pathol. 11:49-56.

9. Lamari, L., and Bernier, C. C. 1991. Genetics of tan necrosis and extensive chlorosis in tan spot of wheat caused by Pyrenophora triticirepentis. Phytopathology 81:1092-1095.

10. Mumford, D. L. 1966. Factors associated with resistance in barley to spot blotch. Phytopathology 56:79-82.

11. Nutter, F. W., Jr., and Pederson, V. D. 1984. Barley seedling disease reaction not a good predictor of adult plant reaction to spot blotch (Helminthosporium sativum). (Abstr.) Phytopathology 74:1270-1271.

12. Nutter, F. W., Jr., Pederson, V. D., and Foster, A. E. 1985. Effect of inoculations with Cochliobolus sativus at specific growth stages on grain yield and quality of malting barley. Crop Sci. 25:933-938.

13. Pringle, R. B. 1979. Role of toxins in etiology of spot blotch disease of barley. Can. Plant Dis. Surv. 59:74-79.

14. Steffenson, B. J. 1997. Spot blotch. Pages 3536 in: Compendium of Barley Diseases. 2nd ed. D. E. Mathre, ed. American Phytopathological Society, St. Paul, MN.

15. Steffenson, B. J., and Fetch, T. G., Jr. 1996. Evaluation of barley for resistance to spot blotch, 1995. Biol. Cult. Tests Control Plant Dis. 11:79.

16. Steffenson, B. J., Fetch, T. G., Jr., Wesenberg, D., and Bockelman, H. 1998. Sources of resistance to net and spot blotch in barley. (Abstr.) Phytopathology 88:S85.

17. Steffenson, B. J., Hayes, P. M., and Kleinhofs, A. 1996. Genetics of seedling and adult plant resistance to net blotch (Pyrenophora teres $\mathrm{f}$. teres) and spot blotch (Cochliobolus sativus) in barley. Theor. Appl. Genet. 92:552-558.

18. Tekauz, A. 1985. A numerical scale to classify reactions of barley to Pyrenophora teres. Can. J. Plant Pathol. 7:181-183.

19. Tomás, A., and Bockus, W. W. 1987. Cultivarspecific toxicity of culture filtrates of Pyrenophora tritici-repentis. Phytopathology 77:1337-1340.

20. Tuite, J. 1969. Plant Pathological Methods. Burgess Publishing Co., Minneapolis, MN.

21. Valjavec-Gratian, M. 1996. Genetics of resistance and virulence in the Hordeum vulgare:Cochliobolus sativus pathosystem. Ph.D. diss. North Dakota State University, Fargo.

22. Valjavec-Gratian, M., and Steffenson, B. J. 1997. Genetics of virulence in Cochliobolus sativus and resistance in barley. Phytopathology 87:1140-1143.

23. Valjavec-Gratian, M., and Steffenson, B. J. 1997. Pathotypes of Cochliobolus sativus on barley in North Dakota. Plant Dis. 81:12751278.

24. Zadoks, J. C., Chang, T. T., and Konzak, C. F 1974. A decimal code for the growth stages of cereals. Weed Res. 14:415-421. 